\title{
Astronomy: learning theories applicable for education in planetarium environment.
}

\author{
Nataliya Kovalenko ${ }^{1,2,3}$ \\ ${ }^{1}$ Astronomical Observatory of Kyiv National University, 04053 Observatorna str.3, Kyiv, Ukraine \\ ${ }^{2}$ Strömstad Academy, SE-45280 Strömstad, Sweden \\ ${ }^{3}$ Kyiv Planetarium, 03150, Velyka Vasylkivska 57/3, Kyiv, Ukraine
}

\begin{abstract}
How do people learn in general and study astronomy in particular? To develop a coherent educational policy we need an appropriate theory. Does learning consist of the incremental addition of individual "bits" of information into the mind? Or is learning an active process that transforms the mind of the learner? Among different theories on how people learn are: Behaviorism, Neuroscience, Right Brain vs. Left Brain, Communities of Practice, Control Theory, Observational Learning (Social learning theory), Vygotsky and Social Cognition, Learning Styles, Piaget's theory, Constructivism, Brain-based Learning, Multiple Intelligences. These theories are described in brief. All of the above mentioned learning theories may be applicable to some extent in the case of astronomy education in a planetarium environment. Especially the Multiple Intelligences theory can be tested perfectly while teaching in Planetarium and thus should be taken into more thorough consideration. It is discussed what a planetarium may offer to the audience with different types of intelligences, according to the Multiple Intelligences approach.
\end{abstract}

\section{Theories of learning}

A theory of learning strives for a fairly clear notion of how people learn. Without appropriate theory it is not possible to develop a coherent educational policy. Does learning consist of the incremental addition of individual "bits" of information into the mind? Or is learning an active process that transforms the mind of the learner?

Educators' views on learning theory will determine the focus of their attention. Acceptance of a transmission-absorption view of learning requires placing major focus on the presentation of the subject in units appropriate for learning. In contrast, active, developmental learning theories require that much more attention be paid to the learner.

If learning is an active process, determined by the individual, then what are the characteristics of learners? Presumably, learners of different types (for example, Gardner's Multiple Intelligences Learning Theory assigns such types as Linguistic, Musical, Logical/Mathematical, Spatial, Bodily/Kinesthetic, Intrapersonal, Interpersonal, for reference please see [7]) use their preferred style independent of the subject that is to be learned, although certain kinds of learning activities lend themselves more readily to one 
learning style or another. Among different theories on how people learn are (please consult [21]):

Behaviorism is a theory of animal and human learning that only focuses on objectively observable behaviors and discounts mental activities. Behavior theorists define learning as nothing more than the acquisition of new behavior.

Neuroscience is the study of the human nervous system, the brain, and the biological basis of consciousness, perception, memory, and learning. Neuroscience links our observations about cognitive behavior with the actual physical processes that support such behavior. This theory is still "young" and is undergoing rapid, controversial development.

Right Brain vs. Left Brain - this theory of the structure and functions of the mind suggests that the two different sides of the brain control two different "modes" of thinking. Left-brain scholastic subjects focus on logical thinking, analysis, and accuracy. Rightbrained subjects, on the other hand, focus on aesthetics, feeling, and creativity. The theory also suggests that each of us prefers one mode over the other.

Communities of Practice - this approach views learning as an act of membership in a "community of practice." The theory seeks to understand both the structure of communities and how learning occurs in them. Knowledge is inseparable from practice. It is not possible to know without doing. By doing, we learn.

Control Theory - this theory of motivation proposed by William Glasser [8] contends that behavior is never caused by a response to an outside stimulus. Instead, the control theory states that behavior is inspired by what a person wants most at any given time: survival, love, power, freedom, or any other basic human need.

Observational Learning, also called social learning theory, occurs when an observer's behavior changes after viewing the behavior of a model. An observer's behavior can be affected by the positive or negative consequences - called vicarious reinforcement or vicarious punishment - of a model's behavior.

Vygotsky and Social Cognition - this learning model asserts that culture is the prime determinant of individual development. Humans are the only species to have created culture, and every human child develops in the context of a culture. Therefore, a child's learning development is affected in ways large and small by the culture - including the culture of family environment - in which he or she is enmeshed.

Learning Styles - this approach to learning emphasizes the fact that individuals perceive and process information in very different ways. The learning styles theory implies that how much individuals learn has more to do with whether the educational experience is geared toward their particular style of learning than whether or not they are "smart." In fact, educators should not ask, "Is this student smart?" but rather "How is this student smart?"

Different individuals have a tendency to both perceive and process information differently. The different ways of doing so are generally classified as:

- Concrete and abstract perceivers. Concrete perceivers absorb information through direct experience, by doing, acting, sensing, and feeling. Abstract perceivers, however, take in information through analysis, observation, and thinking.

- Active and reflective processors. Active processors make sense of an experience by immediately using the new information. Reflective processors make sense of an experience by reflecting on and thinking about it.

Piaget's theory (Swiss biologist and psychologist, 1896-1980) is based on the idea that the developing child builds cognitive structures--in other words, mental "maps," schemes, or networked concepts for understanding and responding to physical experiences within his or her environment. Piaget further attested that a child's cognitive structure increases in sophistication with development, moving from a few innate reflexes such as crying and sucking to highly complex mental activities. 
Piaget's theory identifies four developmental stages and the processes by which children progress through them. The four stages are: Sensorimotor stage (birth - 2 years old), Preoperational stage (ages 2-7), Concrete operations (ages 7-11), Formal operations (beginning at ages 11-15).

During all development stages, the child experiences his or her environment using whatever mental maps he or she has constructed so far. If the experience is different or new, the child loses equilibrium, and alters his or her cognitive structure to accommodate the new conditions. This way, the child erects more and more adequate cognitive structures.

Constructivism is a philosophy of learning founded on the premise that, by reflecting on our experiences, we construct our own understanding of the world we live in. Each of us generates our own "rules" and "mental models," which we use to make sense of our experiences. Learning, therefore, is simply the process of adjusting our mental models to accommodate new experiences.

There are several guiding principles of constructivism:

1.Learning is a search for meaning. Therefore, learning must start with the issues around which students are actively trying to construct meaning.

2. Meaning requires understanding wholes as well as parts. And parts must be understood in the context of wholes. Therefore, the learning process focuses on primary concepts, not isolated facts.

3. In order to teach well, we must understand the mental models that students use to perceive the world and the assumptions they make to support those models.

4. The purpose of learning is for an individual to construct his or her own meaning, not just memorize the "right" answers and regurgitate someone else's meaning. Since education is inherently interdisciplinary, the only valuable way to measure learning is to make the assessment part of the learning process, ensuring it provides students with information on the quality of their learning.

Constructivism calls for the elimination of a standardized curriculum. Instead, it promotes using curricula customized to the students' prior knowledge. Also, it emphasizes hands-on problem solving. Assessment becomes part of the learning process so that students play a larger role in judging their own progress.

Under the theory of constructivism, educators focus on making connections between facts and fostering new understanding in students. Instructors tailor their teaching strategies to student responses and encourage students to analyze, interpret, and predict information. Teachers also rely heavily on open-ended questions and promote extensive dialogue among students.

Brain-based Learning - is a comprehensive approach to instruction using current research from neuroscience. Brain-based education emphasizes how the brain learns naturally and is based on what we currently know about the actual structure and function of the human brain at varying developmental stages. Using the latest neural research, educational techniques that are brain friendly provide a biologically driven framework for creating effective instruction. This theory also helps to explain recurring learning behaviors, and is a meta-concept that includes an eclectic mix of techniques. Currently, related techniques stress allowing teachers to connect learning to students' real lives and emotional experiences, as well as their personal histories and experiences. This form of learning also encompasses such newer educational concepts like:

- mastery learning,

- experiential learning,

- learning styles,

- multiple intelligences,

- cooperative learning, 
- practical simulations,

- problem-based learning,

- movement education.

The three instructional techniques associated with brain-based learning are:

1. Orchestrated immersion--Creating learning environments that fully immerse students in an educational experience;

2. Relaxed alertness--Trying to eliminate fear in learners, while maintaining a highly challenging environment;

3. Active processing--Allowing the learner to consolidate and internalize information by actively processing it.

Core principles directing brain-based education, specified by Caine and Caine [4], are:

- The brain is a parallel processor. It can perform several activities at once.

- The brain perceives wholes and parts simultaneously.

- Information is stored in multiple areas of the brain, and can be retrieved through multiple memory and neural pathways.

- Learning engages the whole body. All learning is mind-body: movement, foods, attention cycles, and chemicals modulate learning.

- Humans search for meaning is innate.

- The search for meaning comes through patterning.

- Emotions are critical to patterning, and drive our attention, meaning and memory.

- Meaning is more important than just information.

- Learning involves focused attention and peripheral perception.

- We have two types of memory: spatial and rote.

- We understand best when facts are embedded in natural spatial memory.

- The brain is social. It develops better in concert with other brains.

- Complex learning is enhanced by challenge and inhibited by stress.

- Every brain in uniquely organized.

- Learning is developmental.

Utilizing both music and art:

One of the key tenets of brain-based education is that attention follows emotion, and both music and art often tap into the emotional areas and thus are natural conduits for remembering and connecting information.

Music: Music can lower stress, boost learning when used 3 different ways:

- as a carrier - using melody or beat to encode content,

- as arousal - to calm down or energize,

- as a primer - to prepare specific pathways for learning content, impacts the immune system, and is an energy source for the brain.

Art: Art is an important part of brain-based education in that it provides many learners with avenues of expression and emotional connection and release. It is important at many levels. For instance, it is important in technology in order to create aesthetically pleasing PowerPoint presentations and multi-media displays that showcase work and make the information and facts presented memorable. Art can be metaphoric creating simple icons or images that ground larger more complex ideas. Art has diverse power and inherent potential to create deep emotional connections and aid in memory retrieval.

According to William Glasser [8],

We learn....

$10 \%$ of what we read. 
$20 \%$ of what we hear.

$30 \%$ of what we see.

$50 \%$ of what we both see and hear.

$70 \%$ of what is discussed with others

$80 \%$ of what we experience

$95 \%$ of what we teach

Multiple Intelligences - this theory of human intelligence, developed by psychologist Howard Gardner, suggests there are at least seven ways that people have of perceiving and understanding the world. Gardner [7] labels each of these ways a distinct "intelligence" - in other words, a set of skills allowing individuals to find and resolve genuine problems they face.

While Gardner suggests his list of intelligences may not be exhaustive, he identifies the following eight:

1.Verbal-Linguistic--The ability to use words and language

2.Logical-Mathematical--The capacity for inductive and deductive thinking and reasoning, as well as the use of numbers and the recognition of abstract patterns

3.Visual-Spatial--The ability to visualize objects and spatial dimensions, and create internal images and pictures

4.Body-Kinesthetic--The wisdom of the body and the ability to control physical motion

5.Musical-Rhythmic--The ability to recognize tonal patterns and sounds, as well as a sensitivity to rhythms and beats

6.Interpersonal--The capacity for person-to-person communications and relationships

7.Intrapersonal--The spiritual, inner states of being, self-reflection, and awareness

8. Naturalistic-Deals with sensing patterns in and making connections to elements in nature.

Traditional schooling heavily favors the verbal-linguistic and logical-mathematical intelligences. Gardner suggests a more balanced curriculum that incorporates the arts, selfawareness, communication, and physical education.

\section{Application of Multiple Intelligences theory of learning in Planetarium environment}

All above mentioned learning theories may be applicable to some extent in the case of the planetarium environment. But to my mind, the two last in the list theories - Brain-based Learning and Multiple Intelligences - should be taken into more thorough consideration and can be tested perfectly while teaching in Planetarium. We use music in 3 ways (as a carrier, arousal, and a primer), involve art, special effects, and full dome projection to make the show emotional and attention catching, create picturesque PowerPoint presentations to make the facts presented memorable, together with our audience we are fulfilling innate search for meaning of observed phenomena, physical processes, and the structure of the Universe. People of different intelligences can experience the content of the Planetarium program in their own way.

Let's consider what a planetarium may offer to the audience with different types of intelligences, according to Multiple Intelligences approach:

Verbal-Linguistic - poetry included in some programs, legends, poetic descriptions of celestial objects and phenomena, quotations from literature in relation to the topic of the show.

Logical-Mathematical - simple calculations, formulae, numbers, some abstract concepts, problem solving activities. 
Visual-Spatial - as some visitors mentioned, feelings of being in the cosmos, perfect support for visualizing space objects and dimensions, the rotating sky above one's head, the movements of celestial objects (e.g. accelerated yearly motion of the Sun, the Moon, the planets, change of the sky aspect for observer at different latitudes, demonstration of eclipses, contours of constellations). The full dome projection system and amazing full dome movies allow visitors to be immersed in the Universe like space and time travellers, feeling themselves as being active participants of an action.

Body-Kinesthetic - for people with this kind of intelligence science museums with interactive exhibits are great fun. Currently in the Kyiv Planetarium we have very few opportunities for visitors with this type of intelligence: a "Lunokhod" mockup, a Buran rocket stylization cash desk, VR glasses and platform, 3 big globes (one of the Earth, one of Venus, and a celestial sphere) that may be rotated, the huge celestial map painted on the wall, and also wall paintings of Zodiac constellations. We used to have landing module "Vostok", a tail segment of a space rocket, a console to communicate with spaceship, but about 10 years ago they were moved by the owner into a specially created museum of cosmonautics at Kyiv Polytechnic Institute. Children have fun while rotating the globes, but we need badly to create interactive exhibition with demonstrations, experiments, measurements, physical experiencing (for example, of being a Moon walker with less gravitation), and interactive kinect-based panels and VR devices like Google expeditions kits. Other opportunities that our planetarium offers for this type of learners are interactive shows like "Astro-Quiz" program [5], live theater and dance performances in synthesis with planetarium effects and full dome projection. Our animators and actors play with children during such theater performances for children, or before the show.

Musical-Rhythmic - in the Planetarium programs we use music and involve sound effects to make the show emotional and attention catching. We run music before and after the show to make visitors feel relaxed. Evening live performances under the dome of Kyiv planetarium, combined with full dome projection or opto-mechanical Zeiss planetarium projection, are of great popularity among our public. These events are called "Jazz under the stars", "Classic music under the stars", "Organ under the stars". Many adult visitors come to planetarium for the first time attracted by these musical shows under the dome and by original combination of live music with starry sky and space visualization.

Interpersonal - the majority of children's programs have characters and scenarios with basic structure - plot, climax, and dénouement. Some programs, in addition to educational meaning, teach children about person-to-person communications and relationships.

Intrapersonal - poetic and philosophical programs describing humanity's place in space and time, personal relation to cosmos. Such audiovisual trips are wellness for the senses allowing time and focus for self-reflection. The Planetarium environment helps a visitor feel a part of the cosmos, forgetting for some time about worries of daily lives on Earth, and induce people to think about eternity.

Naturalistic - Planetarium facilities help to construct the picture of celestial phenomena, to establish connections of observed phenomena with configurations of celestial bodies (explanation of day and night, seasons' change, Moon phases, eclipses, change of sky view during the year).

Thus in contrast with traditional schooling the planetarium environment favors all 8 multiple intelligences and is more balanced.

\section{Acknowledgments}

This material was a part of my science communication Master's Thesis. I appreciate the supervision by Lars Broman and Ernst van Groningen from Dalarna University and Klim Churyumov from Kiev Planetarium. I acknowledge the grant from Armand Spitz Fund for Planetarium Education that helped 
me to recover the study trip to Sweden as distant student of Science Communication Master's Program by Dalarna University. I acknowledge the grant from IAU that allowed me to attend the ISE2A - the International Symposium on Education in Astronomy and Astrobiology.

\section{References}

1. J. Bell. Doing Your Research Project, A guide for first-time researchers in education and social science. Open University Press: Buckingham/Philadelphia (1999)

2. L. Broman. How to write a Master of Science Communication Thesis, Hogskolan Dalarna.

3. L. Broman. Selected papers on Science in Museums, Hogskolan Dalarna.

4. G. Caine, R. Nummela-Caine. Education on the edge of possibility. Alexandria, VA:ASCD-Association for Supervision and Curriculum Development (1997)

5. P. Chastenay. Astro-Quiz. Planetarian June 2006, vol.35, No.2

6. L. Danaia. Common Alternative Astronomical Conceptions Encountered in Junior Secondary Science Classes: Why Is This So? The Astronomy Education Review, Issue 2, Volume 6 (2007)

7. H. Gardner. Frames of Mind: The Theory of Multiple Intelligences. New York: Basic Books (1983)

8. W. Glasser. The Quality School. Harper \& Row (1990)

9. R. R. Hayward. The developing and field testing of an instrument using the planetarium to evaluate the attainment of the concept of annual motion. Presented at the 49th annual meeting of the National Association for Research in Science Teaching, San Francisco (1976)

10. M.-A. Heidmann. The Starry sky. The Planetarium, Revue du Palais de la Decouverte, issue 42 July 1992

11. G. Hein. Learning in the Museum. Routledge: London/ New York (1998)

12. T. Hessel. Justification of a Planetarium, pp.21-22, Planetarian December 2006, vol.35, No.4

13. L. Kelly. Front-end evaluation workshop. Notes from Presentation at the Visitor Centre Stage Action for the Future Conference, Canberra. April 24, 2004

14. http://amol.org.au/evrsig/evrconf.html

15. G. D. Kratzer. A survey of educators' perceptions concerning the impact of the STARLAB planetarium on teaching and learning. McNeese State Univ.independent research project (1997)

16. L. J. Rennie, T. McClafferty. Using visits to interactive science and technology centers, museums, aquaria, and zoos to promote learning in science. Journal of Science Teacher Education, 6 (4): 175-185 (1995)

17. C. Sumners. Quantification of student learning in the museum, planetarium, and IMAX theatre. pp.42-46, 15th International Planetarium Society Conference Proceedings, Montreal (2000)

18. S. A. Vahtera. Time and Space - A Science Education Experiment Based on Activity Learning Education. M.A.Thesis, October 1991, 72p. (29 app.) UNIVERSITY OF HELSINKI, Faculty of Education, Department of Teacher Education

19. M. Webb. Now what do they want? Planetarian June 2006, vol.35, No.2 
20. http://www.astronomynotes.com/chapter1/chindex.htm

21. http://www.funderstanding.com/about_learning.cfm

22. RTDinfo Magazine on European Research, Special issue - November 2005 (http://ec.europa.eu/research/rtdinfo/special_euro/02/article_3150_en.html)

23. Science and Technology: Public Attitudes and Public Understanding http://www.nsf.gov/statistics/seind02/c7/c7s1.htm, http://www.nsf.gov/statistics/seind08/c7/c7s2.htm 